\title{
Patients with Hidradenitis Suppurativa Suffer from Low Health-Related Quality of Life as Measured by the Generic 15D Instrument
}

\author{
Mirkka J. Hirvonen ${ }^{a}$ Rafael Pasternack ${ }^{b}$ Tiina Lipitsäc Armi Vihervaarad \\ Rauno Harvimac Martta Ranta ${ }^{\mathrm{a}}$ Harri Sintonen $^{\mathrm{e}}$ Laura Huilaja $^{\mathrm{f}}$ \\ ${ }^{a}$ AbbVie Oy, Espoo, Finland; ${ }^{\text {b}}$ Department of Dermatology, Tampere University Hospital and Tampere University, \\ Tampere, Finland; 'Department of Dermatology, Kuopio University Hospital and University of Eastern Finland, \\ Kuopio, Finland; dDepartment of Dermatology, Turku University Hospital, Turku, Finland; eDepartment of Public \\ Health, University of Helsinki, Helsinki, Finland; fDepartment of Dermatology and Medical Research Center Oulu, \\ Oulu University Hospital, PEDEGO Research Unit, University of Oulu, Oulu, Finland
}

\section{Keywords}

Acne inversa $\cdot$ Hidradenitis suppurativa $\cdot$ Quality of life . International Hidradenitis Suppurativa Severity Score System · 15D

\begin{abstract}
Introduction: Hidradenitis suppurativa (HS) is a chronic inflammatory skin disease associated with various comorbidities and diminished quality of life (QoL). Among dermatological conditions, HS is reported to most severely diminish QoL. This study aimed to analyse the health-related QoL (HRQoL) of patients with $\mathrm{HS}$ in more detail by using generic to disease-specific HRQoL questionnaires. Correlations between the HRQoL measures and HS disease severity measures were assessed. Methods: We analysed the HRQoL and clinical severity of patients with HS $(N=92)$ treated in 5 Finnish hospitals using HRQoL measurement tools most often used in dermatological clinics, as well as the generic 15D instrument (standardized and self-administered 15-dimensional measure of HRQoL). The disease severity was assessed using the Hurley stage, International Hidradenitis Suppurativa Severity Score System, and disease severity evaluation by the investigator. Results: The mean 15D score of HS pa-
\end{abstract}

karger@karger.com www.karger.com/sad

Karger"

GOPEN ACCESS
C 2021 The Author(s).

Published by S. Karger AG, Basel

This article is licensed under the Creative Commons Attribution 4.0 International License (CC BY) (http://www.karger.com/Services/ OpenAccessLicense). Usage, derivative works and distribution are permitted provided that proper credit is given to the author and the original publisher. tients was low and comparable with that of patients with cancers. No correlation was found between HS severity measures and 15D score, indicating that even mild HS has a high impact on HRQoL. Conclusions: Our findings strengthen the understanding about $\mathrm{HS}$ as a debilitating disease and even compared with non-dermatological conditions and highlight the need of comprehensive care of patients with HS.

(c) 2021 The Author(s).

Published by S. Karger AG, Basel

\section{Introduction}

Hidradenitis suppurativa (HS) is a chronic disease with inflammatory changes in areas with apocrine glands. The disease presents as inflammatory nodules, abscesses, draining tunnels, and scarring [1]. Patients with HS have a heightened risk of several somatic [2] and psychiatric comorbidities [3]. Furthermore, a decreased life span was observed in a Finnish registry-based study [4]. HS is one of the dermatological conditions that most affects patients' quality of life (QoL) [5] and presents with several issues, such as pain and impairment in sex life [6].

An HS-specific QoL questionnaire was recently developed [7]. Until now, the Dermatology Life Quality Index 
(DLQI) was used most to study the QoL of patients with HS [6]; however, the DLQI does not allow the direct comparison between the health-related QoL (HRQoL) effects in dermatological conditions and diseases in other areas. The $15 \mathrm{D}$ instrument is a generic, comprehensive, standardized, and self-administered 15-dimensional measure to assess HRQoL [8] that has been widely used in several areas of medicine, as well as to describe the HRQoL in the general population [9].

In this cross-sectional multicentre study, we analysed the effect of HS on the HRQoL by using both generic, skin disease and HS-specific instruments. The HRQoL of patients with HS was compared with that of age- and sexstandardized patients with psoriasis and the general population. We also studied the correlation of the recently described International Hidradenitis Suppurativa Severity Score System (IHS4) tool [10], Hurley stage, and clinical severity with the HRQoL scores.

\section{Materials and Methods}

\section{Study Population}

The study was conducted in 5 hospital dermatology clinics in Finland. The patients for this study were screened from recent patients with HS in each clinic. A letter of invitation was sent to the patients with the informed consent form. The patients who returned the signed informed consent were then invited on a study visit. On the study visit, the patients were first asked to complete the patient-reported outcome forms. Each patient was interviewed and examined by the investigator. The data from the chart review, interview, and clinical examination were gathered in an electronic case-reporting file system (Viedoc ${ }^{\mathrm{TM}}$, PCG Solutions AB, Uppsala, Sweden). All patients' data on demographics, education, economy, history of HS, smoking, alcohol consumption, comorbidities, and disease severity were collected based on chart review, interview of the patient, and clinical examination. The study protocol was approved by the Regional Ethics Committee of Tampere University Hospital (R16017/2016).

\section{Clinical Evaluation}

At the study visit, the disease severity was evaluated as mild, moderate, or severe (defined as "clinical severity") by an investigator. The number of inflammatory nodules, abscesses, and draining fistulas were counted, and these data were used later to determine the IHS4 [10] total score for each patient, albeit the scoring system was not yet published at the time the study was conducted. The IHS4 was considered mild, moderate, or severe if total scores were $\leq 3,4-10$, or $\geq 11$, respectively [10]. Furthermore, a Hurley severity stage classification [11] was made for each patient.

\section{$15 D$ Instrument}

The generic 15D questionnaire was used to measure HRQoL. The questionnaire comprises the following dimensions: mobility, vision, hearing, breathing, sleeping, eating, speech (communication), excretion, usual activities, mental function, discom- fort and symptoms, depression, distress, vitality, and sexual activity, with 5 ordinal levels on each. The single-index score (15D score), representing the overall HRQoL on a $0-1$ scale $(1=$ full health and $0=$ being dead), and the dimension level values, reflecting the goodness of the levels relative to no problems on the dimension (1) and to being dead (0), are calculated from the questionnaire by using a set of population-based preference or utility weights. The mean dimension-level values are used to draw 15D profiles for groups [8]. The estimated minimum clinically important change or difference in the $15 \mathrm{D}$ score is \pm 0.015 on the basis that people can, on average, feel such a difference [12]. The HRQoL of patients was compared with that of a representative sample of an age- and sex-standardized general population $(N=4,316)$ from the National Health 2011 Health Examination Survey [9] and with that of age- and sex-standardized patients with psoriasis $(N=145)$ from the National Health 2000 Health Examination Survey [13].

\section{Dermatology Life Quality Index}

The DLQI, the most frequently used instrument in randomized controlled trials in dermatology [14], is a simple, validated questionnaire that consists of 10 questions. Each question has 4 possible answers, for a maximum of 3 points, and a total maximum score of 30 . Higher scores indicate more severely affected HRQoL according to the following grade: no effect ( $0-1$ points), small effect ( $2-5$ points), moderate effect (6-10 points), very large effect (11-20 points), and extremely large effect (21-30 points).

\section{Beck Depression Inventory 21}

The Beck Depression Inventory 21 (BDI21) is one of the most widely used instruments for measuring depression symptoms. The questionnaire comprises items relating to depressive, cognitive, and physical symptoms. The BDI21 consists of 21 questions, and each question has 4 possible answers with a value of 0-3 assigned for each answer. Higher total scores indicate more severe depressive symptoms according to the following grade: normal ( $0-12$ points), mild depression (13-18 points), moderate depression (19-29 points), and severe depression ( $\geq 30$ points) [15].

\section{Hidradenitis Suppurativa Symptom Assessment/Hidradenitis}

Suppurativa Impact Assessment

Hidradenitis Suppurativa Symptom Assessment (HSSA) and Hidradenitis Suppurativa Impact Assessment (HSIA) were recently developed to measure signs, symptoms, and impacts of HS, especially for treatment efficacy studies [16]. HSSA consists of 9 questions valued with check boxes from 0 to 10 , where 0 is no symptoms and 10 is very severe symptoms. HSIA consists of 18 questions, 16 with similar scales as in HSSA and 2 questions assessing presence and absence from work and school.

\section{Statistical Analyses}

The descriptive statistics of patient characteristics are presented as means and standard deviations (SDs) and percentages. Pearson correlations were calculated between the scores of different instruments. Statistical significance of the differences between the groups in the means of variables was tested with independent samples $t$ test. $p<0.05$ was considered statistically significant. The analyses were conducted using SPSS Statistics, version 25.0 (IBM, Armonk, NY, USA). 
Table 1. Demographics, lifestyle characteristics, disease, and HRQoL assessments of patients with HS $(N=92)$

\begin{tabular}{|c|c|c|c|}
\hline & Total & Female & Male \\
\hline \multicolumn{4}{|l|}{ Demographic, mean \pm SD } \\
\hline Age, years & $42.2 \pm 13.1$ & $41.4 \pm 11.1$ & $43.2 \pm 15.2$ \\
\hline Age at onset $(N=88)^{\mathrm{a}}$, years & $25.2 \pm 11.7$ & $23.8 \pm 9.9$ & $27.1 \pm 13.6$ \\
\hline Age at diagnosis, years & $37.4 \pm 12.7$ & $36.2 \pm 10.8$ & $39.0 \pm 14.6$ \\
\hline Diagnostic delay $(N=88)^{a}$, years & $12.8 \pm 10.7$ & $13.0 \pm 10.4$ & $12.5 \pm 11.2$ \\
\hline BMI & $32.9 \pm 5.5$ & $32.8 \pm 5.9$ & $33.1 \pm 5.2$ \\
\hline \multicolumn{4}{|l|}{ Lifestyle, $n / N(\%)$} \\
\hline Current smokers & $53 / 92(57.6)$ & $24 / 51(47.1)$ & 29/41 (70.7) \\
\hline Never smoked & $15 / 92(16.3)$ & $13 / 51(25.5)$ & 2/41 (4.9) \\
\hline Smoking at diagnosis & $67 / 92(72.8)$ & $34 / 51(66.7)$ & $33 / 41(80.5)$ \\
\hline Mean weekly alcohol consumption, mean \pm SD, g & $55.5 \pm 102.0$ & $38.0 \pm 90.6$ & $77.2 \pm 111.9$ \\
\hline Median weekly alcohol consumption, g & 12.0 & 6.0 & 48.0 \\
\hline \multicolumn{4}{|l|}{ Assessment tool, mean \pm SD } \\
\hline $15 \mathrm{D}(N=89)^{\mathrm{b}}$ & $0.863 \pm 0.091$ & $0.857 \pm 0.083$ & $0.871 \pm 0.102$ \\
\hline DLQI & $7.50 \pm 6.18$ & $7.90 \pm 6.02$ & $7.00 \pm 6.42$ \\
\hline $\mathrm{BDI} 21$ & $8.97 \pm 7.66$ & $9.69 \pm 8.17$ & $8.07 \pm 6.97$ \\
\hline HSSA & $3.81 \pm 2.64$ & $3.67 \pm 2.56$ & $3.99 \pm 2.75$ \\
\hline HSIA & $2.80 \pm 2.44$ & $2.75 \pm 2.36$ & $2.85 \pm 2.56$ \\
\hline $\mathrm{IHS} 4$, mean \pm SD & $7.6 \pm 8.8$ & $6.7 \pm 7.0$ & $8.6 \pm 10.6$ \\
\hline \multicolumn{4}{|l|}{ Clinical severity, $n(\%)$} \\
\hline Mild & $44(47.8)$ & $24(47.1)$ & $20(48.8)$ \\
\hline Moderate & $38(41.3)$ & $23(45.1)$ & $15(36.6)$ \\
\hline Severe & $10(10.9)$ & $4(7.8)$ & $6(14.6)$ \\
\hline \multicolumn{4}{|l|}{ Hurley stage, $n(\%)$} \\
\hline 1 & $16(17.4)$ & $9(17.6)$ & $7(17.1)$ \\
\hline 2 & $62(67.4)$ & $36(70.6)$ & $26(63.4)$ \\
\hline 3 & $14(15.2)$ & $6(11.8)$ & $8(19.5)$ \\
\hline
\end{tabular}

15D, 15-dimensional questionnaire; BDI21, Beck Depression Inventory 21; BMI, body mass index; DLQI, Dermatology Life Quality Index; HS, hidradenitis suppurativa; HSSA, Hidradenitis Suppurativa Symptom Assessment; HSIA, Hidradenitis Suppurativa Impact Assessment; IHS4, International Hidradenitis Suppurativa Severity Score System; SD, standard deviation. ${ }^{a}$ Data available for 88 patients. ${ }^{b}$ Data available for 89 patients.

Table 2. Comorbidities in study population

\begin{tabular}{llll}
\hline Comorbidity & $\begin{array}{l}\text { Total } \\
(N=92), n(\%)\end{array}$ & $\begin{array}{l}\text { Female } \\
(N=51), n(\%)\end{array}$ & $\begin{array}{l}\text { Male } \\
(N=41), n(\%)\end{array}$ \\
\hline Obesity & $40(43.5)$ & $24(47.1)$ & $16(39.0)$ \\
Cardiovascular disease & $29(31.5)$ & $12(23.5)$ & $17(41.5)$ \\
Skin condition & $24(26.1)$ & $15(29.4)$ & $9(22.0)$ \\
Other & $21(22.8)$ & $17(33.3)$ & $4(9.8)$ \\
Neurological disease & $18(19.6)$ & $10(19.6)$ & $8(19.5)$ \\
Psychiatric disorder & $17(18.5)$ & $12(23.5)$ & $5(12.2)$ \\
Diabetes & $16(17.4)$ & $8(15.7)$ & $8(19.5)$ \\
Sleep disorder & $16(17.4)$ & $7(13.7)$ & $9(22.0)$ \\
Respiratory disease & $16(17.4)$ & $10(19.6)$ & $6(14.6)$ \\
Metabolic disease and hormonal disorder & $13(14.1)$ & $6(11.8)$ & $7(17.1)$ \\
Joint disorder and musculoskeletal disease & $11(12.0)$ & $8(15.7)$ & $3(7.3)$ \\
Inflammatory bowel disease & $3(3.3)$ & $3(5.9)$ & $0(0.0)$ \\
Malignant disease & $1(1.1)$ & $0(0.0)$ & $1(2.4)$ \\
Autoimmune disease & $1(1.1)$ & $0(0.0)$ & $1(2.4)$ \\
\hline
\end{tabular}

Quality of Life in Hidradenitis

Suppurativa
Skin Appendage Disord 2022;8:221-227 DOI: $10.1159 / 000520839$ 


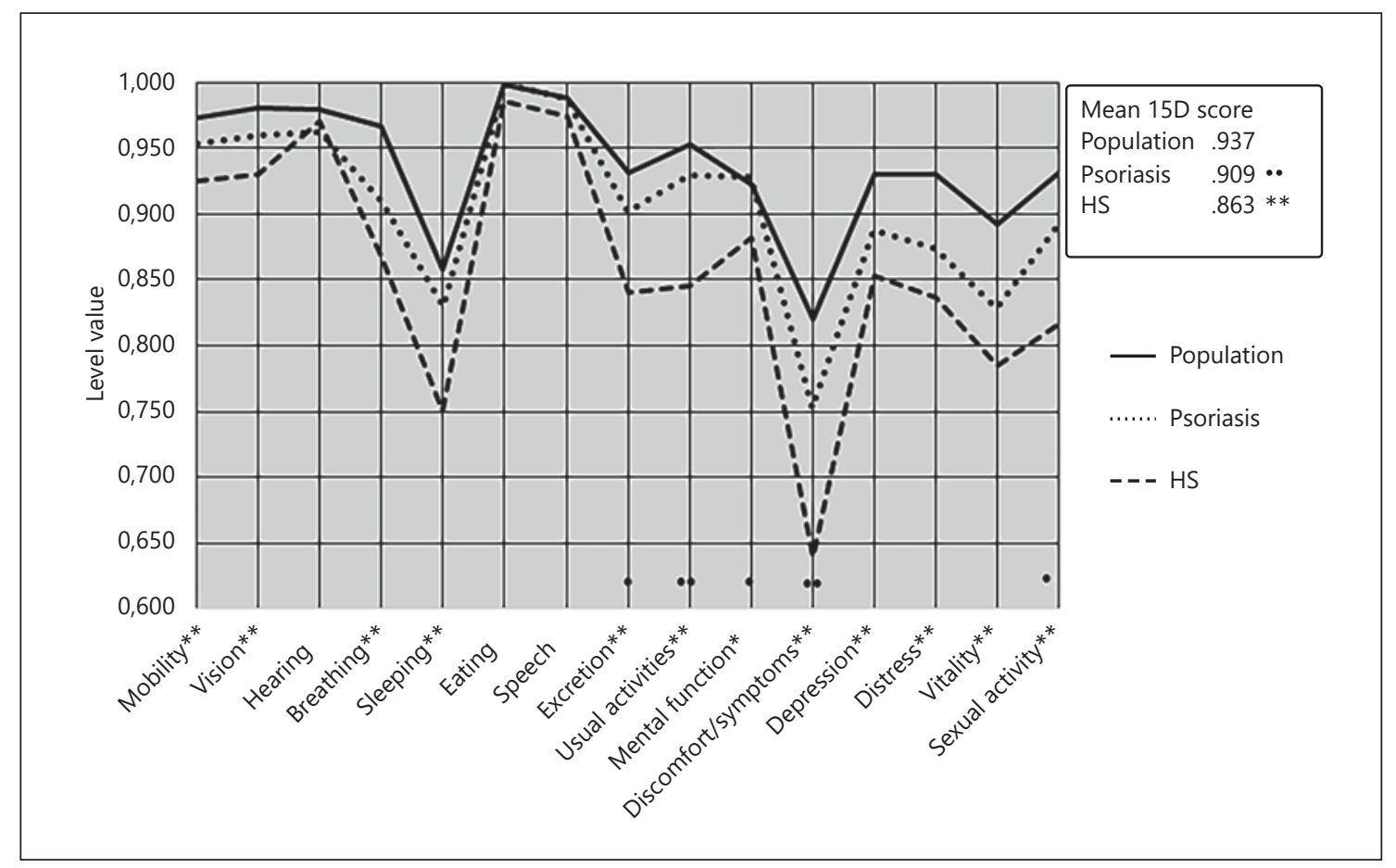

Fig. 1. Mean 15D scores and profiles of patients with HS $(N=89)$, age- and sex-standardized cohorts of the Finnish general population $(N=4,316)$ [9], and patients with psoriasis $(N=145)$ [13]. Statistical significance of the difference in the means between the general population and patients with HS: ${ }^{*} p<0.05,{ }^{* *} p<0.01$. Statistical significance of difference in the means between patients with psoriasis and those with HS: $\bullet p<0.05, \bullet \bullet p<0.01$. 15D, 15-dimensional questionnaire; HS, hidradenitis suppurativa.

Table 3. Pearson correlations between the scores of different instruments

\begin{tabular}{|c|c|c|c|c|c|c|c|c|}
\hline $15 \mathrm{D}$ & 1 & & & & & & & \\
\hline BDI21 & $-0.727^{* *}$ & $0.517^{* *}$ & 1 & & & & & \\
\hline HSSA & $-0.303^{* *}$ & $0.780^{* *}$ & $0.329^{* *}$ & 1 & & & & \\
\hline HSIA & $-0.517^{* *}$ & $0.902^{* *}$ & $0.519^{* *}$ & $0.856^{* *}$ & 1 & & & \\
\hline Clinical severity & $-0.212^{*}$ & $0.500^{* *}$ & 0.149 & $0.456^{* *}$ & $0.437^{* *}$ & 1 & & \\
\hline IHS4 & -0.103 & $0.355^{* *}$ & -0.008 & $0.373^{* *}$ & $0.322^{* *}$ & $0.637^{* *}$ & $0.420^{* *}$ & 1 \\
\hline
\end{tabular}

15D, 15-dimensional questionnaire; BDI21, Beck Depression Inventory 21; DLQI, Dermatology Life Quality Index; HSSA, Hidradenitis Suppurativa Symptom Assessment; HSIA, Hidradenitis Suppurativa Impact Assessment; IHS4, International Hidradenitis Suppurativa Severity Score System. ${ }^{* *}$ Correlation is significant at the 0.01 level (2-tailed). * Correlation is significant at the 0.05 level (2-tailed).

\section{Results}

Demographics and lifestyle data of the 92 patients included are presented in Table 1. Obesity and cardiovascular diseases were the most often found comorbidities (Table 2). The mean (SD) 15D score of patients with HS was 0.863 (0.091) (Table 1; Fig. 1), lower than that of the age- and sex-standardized population $0.937(0.016)$ and that of age- and sex-standardized patients with psoriasis 0.909 (0.082) (Fig. 1). These differences between the groups are statistically significant $(p<0.01)$ and clinically important. Only $2.2 \%(2 / 89)$ of patients obtained a 15D 
score of 1.0 suggesting full health. Apart from hearing, eating, and speech (communication), patients with HS were statistically significantly worse off than the age- and sex-standardized population on all 15D dimensions. The biggest absolute differences were seen on the dimensions of sleeping, excretion, usual activities, and discomfort and symptoms, as well as on the psychical dimensions. Compared with age- and sex-standardized patients with psoriasis, those with HS were statistically significantly worse on the dimensions of excretion, usual activities, mental function, discomfort and symptoms, and sexual activity (Fig. 1).

The 15D scores had an especially high correlation with the BDI21 scores, a high correlation with the DLQI and HSIA scores, and a somewhat lower correlation with the HSSA scores. There was no statistically significant correlation with the IHS4 scores and Hurley stage. The HSSA and HSIA scores correlated very strongly between each other and with the DLQI scores. The correlation of IHS4 was strong with the clinical severity assessed by the physician (Table 3).

\section{Discussion}

The QoL in patients with HS has mostly been studied by using the skin disease-specific DLQI, which allows comparison with other skin diseases but does not allow comparison with other chronic and non-chronic states, healthy control populations [17], or quality-adjusted life year-based health economic evaluation of different interventions. The use of generic HRQoL tools thus far has been sparse in HS studies [17]. Although the 15D has been previously used in a small population of 26 patients with HS [18], our study, to the best of our knowledge, is the first one to describe the correlations of this generic instrument with other HRQoL measures and disease severity measures in HS disease. In our study, patients with HS had a $15 \mathrm{D}$ total score even lower $(0.863)$ than previously described $(0.882 \pm 0.083[18])$; the score corresponds to total scores in diseases such as prostate cancer (0.897 and 0.855 for locally advanced and metastatic, respectively [19]), metastatic breast cancer (0.82 [20]), inflammatory bowel disease (0.874 [21]), and axial spondyloarthritis (0.85 [22]). However, comparisons should be made with caution because the sex and age distribution in these populations may differ from our population.

The presence of current depressive symptoms has been reported to markedly reduce HRQoL as measured by $15 \mathrm{D}$, e.g., in primary care patients [23]. The mean
BDI21 score in our population was 8.97 (SD 7.66) corresponding to "no depression." However, up to $13.1 \%$ of these cases had BDI21 scores of 19-63, denoting moderate to severe signs of depression, and $16.3 \%$ had BDI21 scores of 13-18, denoting mild signs of depression. Also in our population, the $15 \mathrm{D}$ scores had a high correlation with the BDI21 scores (Table 3). In addition, as many as $18.5 \%$ (17/92) of our patients had diagnosed psychiatric comorbidity (including depression and anxiety), which is comparable with findings from the studies from Finnish national registries [3].

As in previous studies, we found no relationship for the generic HRQoL instrument with the severity measures of HS, including Hurley staging and the IHS4 [24]. In our study, the mean DLQI score was 7.50 (SD 6.18), indicating moderate effects in QoL. We found a weak correlation of the DLQI with Hurley staging and IHS4 disease severity measures, while correlation was moderate with the clinical severity. These findings are in line with previous reports $[10,17]$.

The strength of our study is that all the study subjects were carefully interviewed and examined by a dermatologist. In addition, several different methods were used to measure the HRQoL. Although our population was based on patients treated in a hospital, we assume that the study population well represents the majority of patients diagnosed with HS because in Finland, most patients with HS are treated in public health care. Patients treated by private dermatologists are a small minority of all patients and do not markedly deviate from patients treated in hospital policlinics.

HS is one of the most debilitating skin diseases $[25,26]$. With the use of the generic 15D instrument, we show in this study that HS has a remarkable effect on HRQoL, also when compared with diseases of other disease groups. These effects seem to be independent of HS disease severity as they are present even in mild disease. This finding should raise the understanding of the burden of patients with HS and their need for comprehensive care.

\section{Acknowledgments}

The authors wish to thank all the study investigators and study nurses, including Mari Grönroos, MD, PhD; Ulla Oesch-Lääveri, RN (Department of Dermatology, Päijät-Häme Social and Health Care Group, Lahti, Finland); Leena Koulu, MD, PhD (Department of Dermatology, Turku University Hospital and University of Turku, Turku, Finland); and Tiina Kallio, RN (Department of Dermatology, Tampere University Hospital, Tampere, Finland) for their efforts in conducting the study, as well as all of the patients who participated in the study. Martina Serlachius, PhD (AbbVie 
Oy, Espoo, Finland), is acknowledged for her valuable input in the study design and study initiation. Teppo Huttunen from 4 Pharma is acknowledged for conducting the statistical analyses funded by AbbVie.

\section{Statement of Ethics}

The study protocol was approved by the Regional Ethics Committee of Tampere University Hospital (R16017/2016), and the study was conducted ethically in accordance with the World Medical Association Declaration of Helsinki. Patients for this study were screened from recent patients with HS in each clinic. A letter of invitation was sent to the patients with the informed consent form. The patients who returned the signed informed consent were invited on a study visit.

\section{Conflict of Interest Statement}

Dr Huilaja has received educational grants from CSL Behring, Shire, Janssen-Cilag, Novartis, AbbVie, and LEO Pharma and honoraria from AbbVie, Takeda, Novartis, Sanofi Genzyme, and UCB Pharma for consulting and/or speaking and is an investigator for AbbVie. Dr. Tiina Lipitsä has received educational grants from AbbVie and is an investigator for AbbVie. Dr. Rauno Harvima has received educational grants from AbbVie. Dr. Armi Vihervaara has received educational grants from AbbVie, Janssen, Novartis, and Celgene. Dr. Rafael Pasternack has received educational grants from AbbVie, Janssen, LEO Pharma, Novartis, and Sanofi Genzyme and honoraria from AbbVie, Bristol Myers Squibb, Eli Lilly, Janssen, Novartis, Sanofi Genzyme, and UCB for consulting and/ or speaking; participated in clinical studies sponsored by AbbVie, Novartis, Eli Lilly, and Regeneron; and is an investigator for Abb-
Vie. Harri Sintonen is the developer of the 15D instrument and obtains royalties from its electronic versions. Martta Ranta and Mirkka Hirvonen are employees of AbbVie and may or may not own AbbVie shares. The sponsor of this study, AbbVie, participated in the study design, study research, collection, analysis and interpretation of data and writing, reviewing, and approval of this publication. All authors had access to the data and participated in the development, review, and approval and decision to submit this publication.

\section{Funding Sources}

The design, study conduct, and financial support for the study were provided by AbbVie. AbbVie participated in the interpretation of data, review, and approval of the publication.

\section{Author Contributions}

R.P., M.R., and H.S. contributed to the study design; R.P., T.L., A.V., R.H., and L.H. participated in acquisition of the data. All the authors contributed to analysis of the data. M.J.H., R.P., H.S., and L.H. wrote the first version of the manuscript. All the authors participated in the critical revision of the manuscript and have accepted the final version of the manuscript.

\section{Data Availability Statement}

The data that support the findings of this study are not publicly available due to their containing information that could compromise the privacy of research participants but are available from the corresponding author L.H. upon reasonable request.

\section{References}

1 Zouboulis CC, Desai N, Emtestam L, Hunger RE, Ioannides D, Juhasz I, et al. European S1 guideline for the treatment of hidradenitis suppurativa/acne inversa. J Eur Acad Dermatol Venereol. 2015;29:619-44.

2 Kohorst JJ, Kimball AB, Davis MD. Systemic associations of hidradenitis suppurativa. J Am Acad Dermatol. 2015;73:S27.

3 Huilaja L, Tiri H, Jokelainen J, Timonen M, Tasanen K. Patients with hidradenitis suppurativa have a high psychiatric disease burden: a finnish nationwide registry study. J Invest Dermatol. 2018;138:46-51.

4 Tiri H, Jokelainen J, Timonen M, Tasanen K, Huilaja L. Substantially reduced life expectancy in patients with hidradenitis suppurativa: a Finnish nationwide registry study. Br J Dermatol. 2019;180:1543-4.

5 Balieva F, Kupfer J, Lien L, Gieler U, Finlay AY, Tomas-Aragones L, et al. The burden of common skin diseases assessed with the EQ5D: a European multicentre study in 13 countries. Br J Dermatol. 2017;176:1170-8.
6 Matusiak L. Profound consequences of hidradenitis suppurativa: a review. Br J Dermatol. 2018.

7 Thorlacius L, Esmann S, Miller I, Vinding G, Jemec GBE. Development of HiSQOL: a hidradenitis suppurativa-specific quality of life instrument. Skin Appendage Disord. 2019;5: 221-9.

8 Sintonen H. The 15D instrument of healthrelated quality of life: properties and applications. Ann Med. 2001;33:328-36.

9 Koskinen S, Lundqvist A, Ristiluoma N. Health, functional capacity and welfare in Finland in 2011: National Institute for Health and Welfare (THL); 2012.

10 Zouboulis CC, Tzellos T, Kyrgidis A, Jemec GBE, Bechara FG, Giamarellos-Bourboulis EJ, et al. Development and validation of the international hidradenitis suppurativa severity score system (IHS4), a novel dynamic scoring system to assess HS severity. Br J Dermatol. 2017;177:1401-9.
11 Hurley H, Roenigh RRH. Axillary hyperhidrosis, apocrine bromhidrosis, hidradenitis suppurativa and familial beningn pemhigus: surgical approach. New York: Marcel Dekker; 1989.

12 Alanne S, Roine RP, Räsänen P, Vainiola T, Sintonen $\mathrm{H}$. Estimating the minimum important change in the 15D scores. Qual Life Res. 2015;24:599-606.

13 Aromaa A, Koskinen S. Health and functional capacity in Finland: baseline results of the Health 2000 health examination survey. Helsinki: National Public Health Institute; 2004.

14 Finlay AY, Khan GK. Dermatology life quality index (DLQI): a simple practical measure for routine clinical use. Clin Exp Dermatol. 1994;19:210-6.

15 Beck AT, Ward CH, Mendelson M, Mock J, Erbaugh J. An inventory for measuring depression. Arch Gen Psychiatry. 1961;4:56171. 
16 Kimball AB, Sundaram M, Banderas B, Foley C, Shields AL. Development and initial psychometric evaluation of patient-reported outcome questionnaires to evaluate the symptoms and impact of hidradenitis suppurativa. J Dermatolog Treat. 2018;29:152-64.

17 Chernyshov PV, Zouboulis CC, Tomas-Aragones L, Jemec GB, Svensson A, Manolache L, et al. Quality of life measurement in hidradenitis suppurativa: position statement of the European academy of dermatology and venereology task forces on quality of life and patient-oriented outcomes and acne, rosacea and hidradenitis suppurativa. J Eur Acad Dermatol Venereol. 2019;33:1633-43.

18 Kluger N, Sintonen H, Ranta M, Serlachius M. Health-related quality of life of patients with hidradenitis suppurativa measured with the 15D instrument and comparison with the general population and patients with psoriasis. Skin Appendage Disord. 2018;4:131-5.
19 Bergius S, Torvinen S, Muhonen T, Roine RP, Sintonen $\mathrm{H}$, Taari K. Health-related quality of life among prostate cancer patients: real-life situation at the beginning of treatment. Scand J Urol. 2017;51:13-9.

20 Rautalin M, Farkkila N, Sintonen H, Saarto T, Taari K, Jahkola T, et al. Health-related quality of life in different states of breast cancer: comparing different instruments. Acta Oncol. 2018;57:622-8.

21 Haapamaki J, Roine RP, Sintonen $\mathrm{H}, \mathrm{Tu}$ runen U, Farkkila MA, Arkkila PE. Healthrelated quality of life in inflammatory bowel disease measured with the generic 15D instrument. Qual Life Res. 2010;19:919-28.

22 Rohde G, Berg KH, Prøven A, Haugeberg G. The relationship between demographic- and disease-related variables and health-related quality of life in patients with axial spondyloarthritis. BMC Musculoskelet Disord. 2017; 18:328.
23 Riihimäki K, Sintonen H, Vuorilehto M, Jylhä P, Saarni S, Isometsa E. Health-related quality of life of primary care patients with depressive disorders. Eur Psychiatry. 2016;37:28-34.

24 Kolli SS, Senthilnathan A, Cardwell LA, Richardson IM, Feldman SR, Pichardo RO. Hidradenitis suppurativa has an enormous impact on patients' lives. J Am Acad Dermatol. 2020;82:236-8.

25 von der Werth JM, Jemec GB. Morbidity in patients with hidradenitis suppurativa. $\mathrm{Br} J$ Dermatol. 2001;144:809-13.

26 Matusiak Ł, Bieniek A, Szepietowski JC. Hidradenitis suppurativa markedly decreases quality of life and professional activity. J Am Acad Dermatol. 2010;62(8):706708-e1. 